\title{
Stefan Laube
}

\section{Geschäftsinteresse statt ethnischer Identität? Unternehmer von Lódz im 19. Jahrhundert}

Hunderte von qualmenden Schloten gaben Lódz seine eigentümliche Silhouette. Elegante Geschäftsmänner, schmutzige Arbeiter, kaftangekleidete Juden frequentierten die Lódzer Straßen und machten aus der Stadt einen Schmelztiegel orientaler und okzidentaler Lebensweisen. Die Arbeiterschaft lebte unter bedenklichen, zum großen Teil katastrophalen hygienischen Bedingungen in kleinen Einraumwohnungen zusammengepfercht, während die Fabrikanten sich nach historistischen Vorbildern prächtige Paläste erichteten, die meistens von scharfen Hunden bewacht und von hohen Zäunen verdeckt wurden (Strobel 1992: 510f). Menschen, die in Lódz Karriere machten, sahen in der Stadt ein „gelobtes Land“, wo „Milch und Honig fließt“. Andere, die verelendeten, eine „Stätte des Grauens, wo Deutsche und Juden das Regiment führen“ (Bielschowsky 1912: 37).

Die sich in Polen seit den 1870er Jahren herausbildende industrielle Bourgeoisie rekrutierte sich fast ausschließlich aus deutsch- oder jüdischstämmigen Kapitalbesitzern, die ursprünglich auf staatliche Unterstützung angewiesen blieben. Stereotypbildungen waren die Folge: Dem deutschen oder jüdischen Bourgeois und Spekulanten - arrogant und fremd zugleich - stand der fleißige und ehrliche, oft aus dem Bauerntum stammende polnische Handwerker und Arbeiter gegenüber (Kaczynska 1988: 475). Lódz gehörte im 19. Jahrhundert zu Kongresspolen, war zugleich Bestandteil des russischen Zarenreichs und damit einer Herrschaftskonstruktion, die die polnische Teilung bis zum Ersten Weltkrieg zementieren sollte. Der hohe Anteil von Ausländern bei Kaufleuten und Unternehmern gehörte in diesem weiten Wirtschaftsraum zur Tradition (McKay 1970). In Odessa beispielsweise waren es vor allem Juden, aber auch Griechen, Italiener, Franzosen, Belgier, Engländer, Polen und Deutsche, die sich in profitablen Handelsgeschäften hervortaten. In abgeschwächter Form spiegelte sich diese ethnische Vielfalt auch in jeder kongresspolnischen Wirtschaftsmetropole. Nach den Forschungen von Gerschenkron konzentrierte und zentralisierte sich in osteuropäischen Ländern mit verspätet einsetzender und zum großen Teil von ethnischen Minderhei- 
ten getragener Industrialisierung das Kapital besonders schnell und ungehemmt. Die Stadt Lódz verwandelte sich so in einem atemberaubenden Tempo aus einer kleinen dörflichen Siedlung in ein riesiges Industriezentrum. Ende des 19. Jahrhunderts stieg die Einwohnerzahl alle sieben Jahre um 100.000, und Lódz entwickelte sich nach Moskau mit St. Petersburg zum größten Ballungsgebiet der Industriearbeiter im gesamten Zarenreich. Während der Revolution von 1905 war der politische und gewerkschaftliche Organisationsgrad der Arbeiterschaft in Lódz der höchste von Polen und Russland. (Tych 1999: 204). Nachdem sich bis zu Beginn des 19. Jahrhunderts die ethnische Zusammensetzung der kaum tausend Seelen zählenden Bevölkerung auf zwei Drittel Polen und einen Drittel Juden verteilt hatte, sollte sie zwischen 1820 und 1914 um das Sechshundertdreiundzwanzigfache (von 767 auf 478.000) ansteigen und übertraf damit das Bevölkerungswachstum anderer europäischer Textilzentren wie Lyon oder Manchester bei weitem, knapp $80 \%$ waren Polen und Juden, der Rest eingewanderte Deutsche oder deren Nachkommen (Janczak 1982, 38-40).

Mit „Polen“, „Deutschen“ und „Juden“ sind Kategorien der Nationalität gemeint, nach denen seit den statistischen Erhebungen der Jahrhundertwende systematisch gefragt wurde. Vorher war die konfessionelle Verteilung maßgebend gewesen, was immer wieder für Unschärfen sorgen sollte, da nicht jeder Pole katholisch und noch weniger jeder Deutsche evangelisch war. Die Volkszählung von 1897 zeigte, dass die mehr als 300.000 Einwohner zählende Bevölkerung im „Manchester polonais“ (Felde 1935) zu 46\% aus Polen, 29,4\% aus Juden, 21,4\% aus Deutschen und 2,4\% aus Russen bestand. Die Konfessionsstatistik zählte 48\% Bewohner katholischen, 32\% mosaischen, 18\% evangelischen und 2\% russisch-orthodoxen Glaubens (Samus 1999: 13). Die demographische Entwicklung belegt zudem, dass mit wachsender Einwohnerzahl im Laufe der Jahre der Prozentsatz der Polen und Juden anstieg, während der Anteil der Deutschen insbesondere in der Zwischenkriegszeit immer mehr zurückging. In diesem kulturell-ethnischen Grenzraum, wo sich im zaristischen Rahmen deutsche, jüdische und polnische Einflüsse gegenseitig durchdrangen, musste auch die städtische Topographie ethnische Akzente tragen. Die vorwiegend in Industrie und Handwerk beschäftigten Deutschen lebten überproportional südlich des Flüsschen Lodka in den entstehenden Industriesiedlungen der Neustadt, wenig später auch in repräsentativen Gebäuden der Innenstadt entlang der Hauptstraße „ulica Piotrkowska“; die Polen hingegen, die vor allem die Arbeiterschaft stellten, siedelten in den Vorstädten und Randbezirken. Die Juden lebten weitgehend isoliert in der abgeschlossenen Welt des "shtetl“ in Baluty oder Radogoszcz nahe des schon seit dem 14. Jahrhundert bestehenden Ackerbaustädtchen Lódz, d.h. im späteren Nordteil der Industriemetropole (Samus 1999: 25f). 


\section{I.}

Die sich in Abgrenzung zu traditionellen Gesellschaftsmustern in Familie, Religion und Politik herausbildende, aber dennoch stark mit ihnen ambivalent verzahnte kapitalistische Wirtschaftsform findet ein beinahe ideales Forschungsfeld in der mittelpolnischen Textilindustrieregion um Lódz von Mitte des 19. Jahrhunderts bis zum Zweiten Weltkrieg. Weitgehend unabhängig von regionalen Identitäten und ständischen Herrschafts- und Loyalitätsverhältnissen konnte hier eine streng funktional differenzierte, auf vertraglichen Verpflichtungen beruhende Arbeitswelt mit all ihren Möglichkeiten sozialen $\mathrm{Ab}$ - und Aufstiegs entstehen, aus der aber wieder spezifische Formen persönlicher Bindung und Loyalitäten sowie politischer Identitäten und lokalen Patriotismus' hervorgehen sollten.

Die genuine Industrialisierung in Lódz setzte ein bis zwei Jahrzehnte später ein als in Deutschland, verlief dann aber entschieden schneller. Der für zahlreiche osteuropäische Regionen typische insulare Kapitalismus kristallisierte sich im russisch verwalteten Polen vor allem in der Textilindustrieregion um den Lódzer Rayon mit den Städten Pabianice, Zgierz oder Tomaszow. Lódz war zwar zunehmend von Fabriken dominiert, blieb aber ebenso wie die anderen Fabrikstädte eine Enklave in einer Agrargesellschaft mit allen damit verbundenen mentalen Konsequenzen. Rosa Luxemburg war in ihrer Dissertation von 1898 eine der ersten, die den industriellen Wandel in Lódz wissenschaftlich analysierte. Sie grenzte in ihrem historisch-systematischen Ansatz eine Manufakturperiode zwischen 1820 und 1850 von einer Übergangsphase zwischen 1850 und $1870 \mathrm{ab}$, die schließlich in das Zeitalter der Großindustrie gemündet sei. Mit der Großindustrieproduktion war in erster Linie die Baumwollfabrikation gemeint, die das noch weitgehend handwerklich strukturierte Tuchmachergewerbe zunehmend an den Rand drängte. Daneben entwickelten sich mit dem Seiden-, Band- und Trikotagengewerbe lukrative Folgeindustrien. Luxemburg sah in der Lódzer Textilindustrie ein aufgesetztes Unikum, bestehend aus ausländischen Unternehmern, fremdem Kapital, zugewanderten Arbeitskräften und gigantischen Absatzmärkten, an deren Ende im Zeitalter des Hochkapitalismus die völlige Verschmelzung mit dem russischen Wirtschaftsmarkt stehen würde (Luxemburg 1898) und grenzte sich damit von nationalistischen oder patriotischen Deutungen ab, die die Lódzer Industrie als ein Beispiel deutschen Gewerbefleißes und Kulturleistung betrachteten (Bielschowsky, 1912) oder sie bodenständig mit dem polnischen Binnenmarkt verknüpft sahen (Janowicz 1907). Das Beziehungsgeflecht ethnischer Ökonomie formte sich hier in einer ganz bestimmten Art und Weise aus. Wenn auch das Übergewicht im Großbürgertum in Warschau oder Lódz von Kaufleuten und Unternehmern deutscher und jüdischer Herkunft so dominant war, dass man aus polnischer Sicht - wenn es um die ethnische Zugehörigkeit geht - fast von einem geliehenen Status als Großbürger spre- 
chen kann, passten sich die ursprünglich fremden Großbürger doch rasch an und verwandelten sich in ein polnisches Bürgertum (KoLódziejczyk 1986). In Warschau verlief der Assimilationsprozess schneller als in Lódz, wo sich die Unternehmer frühestens in der zweiten, in der Regel jedoch erst in der dritten Generation polonisierten. Hier war in der ausgedehnten Übergangszeit die multinationale Kategorie des „Lódzer Menschen“ präsent, d.h. eines Menschen, der weder ganz deutsch, noch ganz polnisch oder ganz jüdisch eingestellt war, aber stets an seine lohnenden Geschäfte dachte. Der „Lódzer Mensch" stellte ein anthropologisches Spezifikum dar, in dem sich nationalistische Glaubenslehren kaum entfalten konnten. Will man seinen Entstehungsbedingungen auf den Grund gehen, muss man weit in das 19. Jahrhundert zurückblicken.

Ähnlich wie der Große Kurfürst nach dem Dreißigjährigen Krieg mit den Hugenotten Fachkräfte aus dem Ausland nach Brandenburg holte, betrieb nach dem napoleonischen Umbruch die kongresspolnische Regierung nach merkantilen Gesichtspunkten eine einwanderungsfreundliche Politik, um im eigenen Land einen konkurrenzfähigen neuen Industriezweig aufzubauen. Eine großzügige Einwanderungspolitik der drei Teilungsmächte nach 1815 erlaubte jedem Bewohner der verschiedenen Teile Polens, seinen Wohnsitz innerhalb der Teilungsgebiete frei zu wählen. Mit dem Zgierzer Einwanderungsgesetz vom 18.9.1820 wurden in der Wojewodschaft Masowien siebzehn Städte - darunter auch Lódz - zu Fabrikstädten erklärt, da dieser Ort mit einem weit verzweigten System von Wasserläufen, günstigen Verkehrswegen und ausgedehnten Holzbeständen in den nahe gelegenen Wäldern über überaus günstige Voraussetzungen verfügte. Gezielt schickte die Regierung bezahlte Agenten zur Anwerbung geeigneter Handwerker in die gewerbereichen Gebiete des Westens, die sich meist in einer wirtschaftlichen Krisensituation befanden und lockte sie mit einem vielfältigen Angebot von Privilegien und Vergünstigungen nach Polen (Gasiorowska 1922). Die polnische Tuchfabrikation erlebte so in den ersten Jahrzehnten des 19. Jahrhundert ihre erste Blüte. Deutsche halbfertige Produkte gelangten zollfrei nach Kongresspolen, wo sie weiterverarbeitet als polnisches Fabrikat zollfrei nach Russland geliefert werden konnten (Luxemburg 1898: 122). Die administrativen und legislativen Reformen konnten auf den seit 1808 im ehemaligen Großherzogtum Warschau gültigen napoleonischen ,Code Civil' inklusive seines, Code de Commerce ${ }^{6}$ mit seinen industriefreundlichen Bestimmungen zur Gewerbe- und Eigentumsfreiheit aufbauen. Das übrige Zarenreich besaß hingegen noch lange Zeit kein kodifiziertes Zivilrecht, weite Teile Österreichs und Preußens hatten für die Entwicklung einer auf Freiheit und Gleichheit beruhenden bürgerlichen Gesellschaft nur veraltete Bestimmungen. 


\section{II.}

Der vorliegende Beitrag beschäftigt sich mit einer bestimmten sozialen Schicht, die in Lódz über besonders großen Einfluss verfügte. Mit der Industriebourgeoisie hat man eine soziale Gruppe im Blickfeld, mit der bis heute noch zahlreiche Legenden und Mythen in Verbindung gebracht werden. Aus der Sicht polnischer und russischer Journalistik und Historiographie galten deutsche Unternehmer als Vertreter des deutschen Drangs nach Osten, kommunistische Ideologen machten sie zu kapitalistischen Ausbeutern der Arbeiterklasse; auf der anderen Seite glorifizierte die deutsche Seite sie zu Industriepionieren, die den Polen vormachten, wie man ein Industriegebiet aufzubauen hatte. Im Gegensatz zum Denkmodell von Karl Marx, für den der Unternehmer eine austauschbare Figur im Kapitalverwertungsprozess darstellte, könnte man demnach Werner Sombarts Diktum „Der Kapitalismus ist das Werk einzelner hervorragender Männer, daran kann kein Zweifel sein“ (Sombart 1902: 886) am Lódzer Fallbeispiel veranschaulichen.

Nach den jüngsten Berechnungen stellte das Industriebürgertum sowie das Handels- und Finanzbürgertum einschließlich der Familienangehörigen kurz vor 1914 nicht mehr als 2,5\% der Lódzer Einwohnerschaft (Pytlas 1994: 47). Die meisten waren deutscher oder jüdischer Abstammung, wobei der Anteil der Unternehmer deutscher Herkunft zwischen 1860 und 1910 von 63\% auf 44\% sank, die jüdischer Abstammung von 21\% auf 47\% anstieg. Die Deutschen blieben aber dominant bei der Großproduktion von Textilien. Der Startvorteil von Deutschen und Juden in der Unternehmerklasse war für die Polen in dieser Epoche zu groß, sodass sie sich dort nur sehr schüchtern etablieren konnten. Die am Kult der Aufbauleistung der industriellen Pioniere orientierte deutsche Geschichtsschreibung à la Treitschke und besonders Sombart drängte wichtige internationale und sozioökonomische Motivationen in den Hintergrund. Die Migrationswellen aus den deutschen Ländern resultierten aus einer zunehmenden Pauperisierung der dortigen Tuchmacher infolge der Konkurrenz billigerer englischer Maschinenerzeugnisse sowie langandauernder Missernten und Hungerperioden. Zudem machten Zollrestriktionen den Absatz der Textilien in Polen oder Russland weitgehend unmöglich. Die existenzbedrohten Handwerker verbanden die Einwanderung mit der Überwindung der Zollgrenze, um so dem Absatzmarkt zu folgen. Auch ist das große Engagement polnischer Behörden augenscheinlich: Von ihnen gingen die Maßnahmen aus, ausländische Fachkräfte ins Land zu holen, ebenso stellten sie mit günstigen Darlehen Kapital für den neuen Industriezweig parat und gewährten weitere Privilegien, wenn sich jemand entschloss, eine Fabrik zu gründen. Die 1828 nach dem Vorbild der ,Preußischen Seehandlung oder der ,Societé Générale' in Belgien geschaffene ,Polski Bank eröffnete der im Entstehen begriffenen polnischen Industrie dringend benötigte Kreditmöglichkeiten. Durchaus virulente ethnische Identitätsbildungen 
in der Lódzer Unternehmerschaft rückten immer wieder in den Hintergrund, wenn es um konkrete Wirtschaftsinteressen geht. Viel stärker beeinflussten zoll-, verkehrs und marktpolitische Voraussetzungen unternehmerische Entscheidungen. Nach dem in ganz Europa Sympathien hervorrufenden polnischen Aufstand von 1830 und 1831 baute das Zarenregime als politische Sanktion zwischen Russland und Kongresspolen eine Zollmauer auf. Nicht zuletzt deswegen, aber auch weil Naturalwirtschaft, Leibeigenschaft und fehlende Verkehrswege sowie eine auf Handarbeit beruhende Textilindustrie ohne Dampfkraft dem Ausbau des polnischen Marktes enge Grenzen setzten, ging die Produktion spürbar zurück. Erst mit der Abschaffung der Zollbarrieren zwischen Russland und Polen im Jahre 1851 wurden die Voraussetzungen zur Schaffung der Fabrikindustrie geschaffen, da man nun für große Massen produzieren konnte. Der riesige Absatzmarkt, der bis zum Eismeer und über den Kaukasus bis zum Chinesischen Meer reichte, glich die Nachteile mangelnder Kaufkraft aus. Gerade der verlustreiche Krimkrieg mit der Blockade der Seegrenzen verursachte in Russland eine Massennachfrage der russischen Armee nach Textilien, die in erster Linie von der Lódzer Industrie befriedigt werden konnte. Dem Bau jeder Trasse nach Russland - 1870 entstand eine Eisenbahnverbindung mit Moskau, 1871 mit Kiew - folgte eine verstärkte Nachfrage nach polnischen Textilprodukten. Im Zeitalter der etablierten Fabrikindustrie existierte seit 1877 hingegen wieder ein protektionistisches System, das aber diesmal die Entwicklung zum Hochkapitalismus massiv unterstützte, in dem es sie vor ausländischer Konkurrenz schützte.

„Auf diese Weise, nachdem in den Jahren 1860-1877 alle Hauptbedingungen der industriellen Entwicklung - ein innerer Markt, Verkehrsmittel, eine industrielle Reservearmee - ins Leben gerufen worden waren, schuf die hinzugetretene Zollpolitik eine Treibhausatmosphäre der Monopolpreise, welche die russische und polnische Industrie in ein förmliches Dorado der primitiven kapitalistischen Akkumulation versetzte“ (Luxemburg 1898: 131f.).

Nach der Abschaffung der Leibeigenschaft war die Landwirtschaft auf den Ankauf von Industrieprodukten angewiesen, die zuvor bedarfsgerecht und weitgehend unentgeltlich auf den Gutshöfen hergestellt worden waren. $\mathrm{Zu}-$ gleich verwandelten sich immer mehr Bauern zu Geldbesitzern und damit zu potenziellen Käufern von Fabrikerzeugnissen. Zudem schuf die Proletarisierung breiter Bauernschichten ein Arbeitskräftereservoir für die Fabrikindustrie in Lódz. Zahlreiche große Fabriken sind in Folge der seit 1877 bestehenden restriktiven Zollpolitik entstanden. Der erhobene Goldzoll führte da$\mathrm{zu}$, dass schlesische und sächsische Fabriken ihre Produktion gleich direkt nach Lódz verlegten. Massen verarmter polnischer Bauern und aus Zentralrussland vertriebener jüdischer Kaufleute trafen in Lódz mit deutschen, jüdischen, aber auch französischen Kapitalisten - seit 1879 verlegte die Spinnerei Allart-Rousseau aus Roubaix einen großen Teil ihrer Produktion nach Lódz zusammen. Für eine kleine Schicht von risikofreudigen Unternehmern ver- 
wandelte sich Lódz zwischen 1877 und 1900 angesichts der unbegrenzten Möglichkeiten eines riesigen Absatzmarktes in ein „zweites Amerika, wo das Gold auf der Straße liegen soll“" (Bielschowsky 1912: 37). Die Lódzer Industrie war seit ihrer Entstehung mit dem russischen Absatzmarkt verbunden. Dieser Absatzmarkt stellte die eigentliche Triebfeder der großindustriellen Entwicklung Polens dar. 1892 betrug der Produktionswert der Textilindustrie im Zarenreich über 580 Millionen Rubel, davon stammten knapp ein Fünftel aus Lódz, das sich immer mehr zu einer ernst zu nehmenden Konkurrenz gegenüber St. Petersburg und Moskau entwickelte (Luxemburg 1898: 146). Viele erfolgreiche Lódzer Unternehmen verfügten in Russland - ob nun in Kiew, St. Petersburg, Moskau oder Rostow am Don - über Filialen, die Größten von ihnen bauten sogar in Zentralasien eine eigene Organisation zum Einkauf von Baumwolle auf. In den achtziger Jahren beklagten sich immer mehr Industriekreise aus Moskau über die Güterexpansion aus Lódz (Rose 1918). Unter diesen Rahmenbedingungen musste für die einheimischen Polen die importierte kapitalistische Wirtschaft wie ein „deutscher Schwindel“, wie eine „ausländische giftige Pflanze“ erscheinen, die fremde Unternehmer zu Millionären machte (Luxemburg 1898: 123, Bielschowsky 1912: 32f) und tatsächlich setzte sich der Kapitalismus in Lódz in einer Zeit durch, als die polnische Bevölkerung nach der Niederschlagung des Januaraufstandes von 1864 unter einer schonungslosen Russifizierungspolitik zu leiden hatte, die es den polnischen Schülern sogar verwehrte, in ihrer Muttersprache unterrichtet zu werden. In der Nachkriegszeit, als es für die junge Volksrepublik darum ging, die Anfänge des polnischen Kapitalismus zu bestimmen, brachen immer wieder Kontroversen aus, ob nun in- oder ausländisches Kapital für die rasante Entwicklung der Textilindustrie verantwortlich gewesen sei (z.B. Kula 1955: 56-58). Inzwischen ist es erwiesen, dass bis in die 1870er Jahre kein Kapital aus fremden Staaten in Lódzer Fabriken floss. Das Vermögen der deutschen Großunternehmen basierte auf der Arbeit der Lohnarbeiterschaft und bestand ursprünglich aus Regierungskrediten, dann aus seiner Akkumulation vor Ort sowie der Umwandlung von Handels- in Industriekapital.

\section{III.}

Victors Hugos „Père Madeleine“ aus seinen „Les misérables“ vereinigt Schumpeter vorausgreifend - Unternehmereigenschaften, die auch für jeden Lódzer Fabrikanten Richtschnur des eigenen Handelns hätten darstellen können. Er war Kapitalist und Eigentümer an den Produktionsmitteln, Manager und Risikoträger, Erfinder und organisatorischer Neuerer, Arbeitgeber mit sozialen Verpflichtungen gegenüber den Arbeitern und schließlich einfach Geschäftsmann (Plum 1977: 31, Kocka 1975). Niemand unter den professionellen Großunternehmern in Lódz, wie zum Beispiel - um nur die wichtigsten zu nennen - Ludwik Geyer (1805-1869), der 1837 nach engli- 
schem Vorbild seine Weberei auf Dampfkraft umstellte (Waskiewicz 1958), Karl Wilhelm Scheibler (1820-1881), dessen Baumwollfabrik schon bald ein ganzes Stadtviertel einnahm (Pastula 1994) oder Izrael Poznanki (1833-1900), der zur kleinen Gruppe derjenigen Juden gehörte, denen der Wechsel vom Handel in die Produktion überaus erfolgreich gelang (Gorgolewski 1985), wäre auf den Gedanken gekommen, zu Gunsten ethnischer Rücksichten das Geschäftsinteresse hintenanzustellen. Dem Aufstieg der Polen mit ihrem Selbstverständnis von einer Adels- und Agrarnation in die Schicht der Großindustriellen standen nicht nur mentale Hindernisse im Wege. Erschwert wurde er in Lódz auch dadurch, weil viele deutschstämmige Unternehmer sich nicht nur als Leiter der Produktion, sondern auch des Absatzes verstanden und sich Großkaufleute nur schwer entfalten konnten:

„Der Lódzer Fabrikant ist nicht nur Inhaber der Fabrik, sondern eine Person, die praktisch bis zu den geringsten Kleinigkeiten mit dem Geschäft vertraut ist. Er wohnt in Fabriknähe, beaufsichtigt den gesamten Herstellungshergang und ist überall eingeweiht, sogar dann, wenn bei groBer Ausdehnung des Unternehmens ein Fabrikdirektor vorhanden ist. Er verfolgt genau die Ergebnisse seines Produktionszweigs im Auslande, bestellt, sobald er von neuen, besser und ausgiebiger arbeitenden Maschinen erfährt, diese ohne Bedenken, wenn auch seine bisherigen Maschinen noch in gutem Zustand sind. Die Mehrzahl der Lódzer Fabrikanten verdankt ihre gegenwärtige Lage der eigenen Arbeit." (Bericht der russischen Regierungskommission aus dem Jahre 1886, in: Rogall 1998: 437).

In die zwischen Produzent und Konsument angesiedelte Kaufmannsschicht stiegen zahlreiche Juden auf, die von dort auch zur Tuchmacherei gelangten, da sie es verstanden, neue Erzeugnisse auf den Markt zu bringen und Massenartikel zu lancieren (Bielschowsky 1912: 26). Juden waren geübt im Kleinhandel und in geldwirtschaftlichen Angelegenheiten, besaßen bewegliches Kapital und verfügten oft über internationale Verbindungen (Mosse 1992). Allgemein kann festgestellt werden, dass der jüdische Anteil in der Textilindustrie in dem Maße zunahm, in dem man sich vom Produktionsprozess mit dem Rohstoff entfernte und in der Sphäre des Umgangs mit dem Fertigprodukt bei Handel und Konsum tätig war (Walter 1992). So hatten Juden auch in Lódz, die keineswegs eine homogene Gruppe darstellten, ein deutliches Übergewicht im Handel, im Finanzwesen und im Fuhrgewerbe. Die Juden konnten den Handel deswegen dominieren, weil sie enge Handelsbeziehungen mit ihren Glaubensbrüdern in den Ein- und Ausfuhrländern nutzten und weil sie leicht zugängliche und günstige Kredite gewährten. Insbesondere die so genannten Litwaken, die in den achtziger Jahren in die Stadt strömten, nachdem sie aus den mittelrussischen Gouvernements ausgewiesen worden waren, waren als Agenten und Kleinhändler mit Sprache, Sitte und Bedürfnissen der russischen Bevölkerung vertraut und sollten Lódz mit Russland ökonomisch besonders eng verknüpfen (Friedman 1935).

Abgesehen von den Familien Poznanski, Rappaport und Silberstein beherrschten die Juden keineswegs die Großindustrie, die vorrangig weiterhin aus ortsansässiger, deutschsprachiger Bevölkerung bestand. Deutsche und jü- 
dische Eliten gingen als Repräsentanten ihrer jeweiligen Kulturen pragmatisch miteinander um. Wenn ein jüdischer Garnhändler mit einem deutschen Weber Geschäfte machte, galten Bekenntnisunterschiede wenig (Guesnet 1999: 147). Das Geschäftsinteresse stand im Vordergrund. Auch war für beide die Ambition zunächst noch begrenzt, politisch mitzusprechen, was nicht ohne eine Polonisierung möglich gewesen wäre.

\section{IV.}

Der Lódzer Alltag war von einem vielfältigen geschäftlichen Geflecht geprägt, zwischen deutschen Unternehmern und ihren anfangs auch deutschen Meistern, dann später zwischen polnischen Meistern und polnischen Arbeitern, aber immer auch deutschen und erheblich seltener jüdischen Fabrikarbeitern, zwischen jüdischen Kaufleuten, Handwerkern und Krämern sowie ihren christlichen Geschäftspartnern und Kunden. Gerade der Handel fungierte in Lódz als Schnittstelle unterschiedlicher Lebensformen. Die hiesige Lebenswelt musste in einem hohen Grade modern sein, weil es unter einer Fremdherrschaft nur wenig zentrale Instanzen gab, weil der Führung kirchlicher, schulischer und gesellschaftlicher Einrichtungen auf einem hohen Grad von Eigeninitiative und Selbstverantwortung beruhten und weil man sich im wirtschaftlichen Handeln und kulturellen Leben jeden Tag mit verschiedenen Ethnien und damit verbundenen Lebensweisen auseinander zu setzen hatte. Die im Minderheitenstatus inhärenten religiösen Antriebskräfte - wie von Max Weber immer wieder betont - spielten in Lódz bei der Ausbildung des kapitalistischen Systems nur eine untergeordnete Rolle. Wenn auch der Zusammenhang zwischen protestantischer Ethik im Sinne einer auf Individualismus, Askese und Rationalismus beruhenden Lebensform und dem kapitalistischen Wirtschaftshandeln von modernen Historikern als bestätigt gesehen wird (Kocka 1975: 35ff) und in einer Stadt wie Krefeld zum Beispiel, die seit der Reformation zu einem Refugium von Andersgläubigen wurde, der merkantile Aufschwung der Seidenindustrie den religiösen Außenseitern der Mennoniten, Reformierten und Juden mit ihrem Sinn für das Ökonomisch-Rationale zu verdanken war, gestaltete sich in Lódz dieser Zusammenhang weniger evident. Im Vordergrund stand bei den Migranten des 19. Jahrhunderts von Anfang an nicht religiöse Unterdrückung, sondern wirtschaftliche Not sowie leistungsbereiter Pragmatismus, sich in der Fremde etwas aufzubauen. Die Migrationswellen aus Preußen, Sachsen, dem Rheinland, aus Böhmen und Österreich überschnitten sich im Laufe des 19. Jahrhunderts immer mehr mit den Wanderungsbewegungen der Polen und Juden. Dies bedeutete auch, dass Einwanderer immer wieder nur auf Fremde stießen. Schon Sombart erkannte, dass Gewinn bringende Geschäfte in der Regel nur unter Menschen, die man kaum kennt, zu Stande kommen. Ihm zufolge konnte sich der Unternehmergeist gerade in der Fremde ungehemmt entfalten, da hier keine persönliche Rücksichten genommen werden mussten. 
„Auch das behagliche Sich-Ausleben verbietet sich in der Fremde. Die Fremde ist öde. Sie hat für den Ankömmling keine Seele. Die Umgebung bedeutet ihm nichts ... Es gibt für den Ausgewanderten - das gilt gleichermaßen für den Emigranten wie für den Kolonisten - keine Vergangenheit, es gibt für ihn keine Gegenwart; es gibt für ihn nur eine Zukunft.“ (Sombart 1902: 886).

In Lódz war die Fremde besonders fremd, da hier der Anteil der Einwanderer denjenigen der einheimischen Bevölkerung um ein Vielfaches übertraf.

V.

Die Debatte, wie sich nun die Lódzer Unternehmerschaft zu einer einheitlichen sozialen Formation, die auf Grund ihrer Eigentumsrechte an den Produktionsmitteln identische Klasseninteressen vertraten, entwickelte und wie sich die Klassenbildungsprozesse in Arbeiter- und Unternehmerschaft wechselseitig beeinflussten, ob es eine Klasse der Bourgeoisie gegeben hat, bevor sich die Arbeiterschaft zu einer Klasse formiert hatte und wie sich dazu die Nationsbildung verhielt, ist noch nicht abgeschlossen. Eine intensive Beschäftigung mit dem Lódzer Fall würde bestimmt neue Ergebnisse bringen, auch zu der Frage, was für einen Lódzer Unternehmer nun wichtiger gewesen war: das gemeinsame Klasseninteresse mit anderen Unternehmern oder die gemeinsame, potenziell schichtenübergreifende nationale Zugehörigkeit? Die politische Rhetorik jedenfalls war lange Zeit von der sozialen Konfliktsituation dominiert, wobei nationale und rassische Komponenten auffällig im Hintergrund blieben. Seit den 1890er Jahren wurden in Lódz Kartellbildungen und Unternehmerabsprachen im Hinblick auf das Marktverhalten sichtbar. Die Klasse organisierte sich in Interessenverbänden. Feliks Tych hat herausgefunden, dass während der Revolution von 1905 und 1907 die Arbeiterschaft ob nun polnischer, deutscher oder jüdischer Herkunft - geschlossen gegen die Unternehmerklasse auf die Barrikaden ging. Erst nach ihrem Scheitern hätten sich die Gruppen ethnisch voneinander entfernt und in einer „Symbiose ohne Sympathie“ (Tych 1999: 209) gelebt. Selbst die auf den innerbetrieblichen Erfahrungs- und Handlungsraum bezogene Unternehmerperspektive bliebe unvollständig, wenn sie nicht durch Kategorien der Kultur, Erfahrung und Kommunikation erweitert werden würde, wie dies schon Edward P. Thompson Ende der 60er Jahre vorgeschlagen hat. Denn trotz großer interner Rivalitäten in der Unternehmerklasse muss man von einem Bewusstsein ähnlicher Lebensbedingungen und gemeinsamer Deutungsmuster ausgehen, das sich auch in ihrem Habitus äußerte, wie der Hochachtung vor der individuellen Leistung und dem damit verbundenen Anspruch auf wirtschaftliche Belohnung, soziales Ansehen und politischen Einfluss (Kocka 1987, 43).

Immer wieder fiel auf, dass die Gründer und Leiter der modernen Industrieunternehmen, die häufig auf Grund von Privilegien der Obrigkeiten, d.h. staatlicher Interventionen, von Zwängen der zunftorientierten Wirtschaftsordnung ausgenommen waren, ihr Ansehen keineswegs ausschließlich auf ei- 
gene Leistung und die Marktbedingungen bezogen, sondern eher auf feudale Kriterien der Geburt, des Landbesitzes und des Patriarchalismus. Insbesondere Scheibler zeigte sich in dieser Hinsicht ambitioniert. Der Fabrikant hatte ein Wohnviertel mit 200 Arbeiterhäusern errichtet, fünf Elementarschulen, ein Fabrikhospital, Ambulanzen, eine Apotheke, einen Kinderhort und ein Altersheim. Zudem gab es auf Scheiblers Fabrikgelände Läden, eine Badeanstalt und sogar ein Arbeitsorchester. 1878 erhielt er auf der Pariser Weltausstellung für seine sozialen Experimente eine Goldmedaille. In den Siebzigerjahren führte er eine Krankenhilfskasse sein. Obwohl sich das in Vereinen organisierte Lódzer Wohltätigkeitswesen bis zur Jahrhundertwende in einen jüdischen und einen christlichen Zweig diversifizierte, blieben die karitativen Fabrikeinrichtungen Scheiblers für alle ethnischen Gruppen offen (PietrowEnnker 1999: 120ff). Ebenso wie die Arbeiterschaft entwickelte auch der Unternehmer - in Konfrontation mit den Forderungen des Proletariats - einen Sinn für Vorleistungen in Form industrieller Wohlfahrtspflege, um die Arbeiterschaft sozial einzugliedern (Tanner 1999: 25).

VI.

Noch ziemlich ungeklärt ist die Frage, wie in diesem Erklärungsmodell das immer einflussreichere nationale Prinzip integriert werden könnte. Daher mögen hier am Beispiel von Lódz nur einige Indizien genügen. Als sich die Fabrikarbeiter in den neunziger Jahren in Streikaktionen ihrer Unzufriedenheit Luft machten, reagierten die Lódzer Unternehmer mit der Gründung von Interessenverbänden, wie im Jahre 1906 mit dem „Verband der Lódzer Fabrikanten der Bekleidungsindustrie“ in Berlin. Symptomatisch war dabei, dass sich die Lódzer Unternehmer nach der Ermordung ihres Kollegen Julius Kunitzer während der Revolution von 1905 - Kunitzer galt bei den Arbeitern als schonungsloser Ausbeuter - in klassenmäßiger und nationaler Solidarität in der Hauptstadt des Deutschen Reiches in Sicherheit brachten. Galten überwiegende Teile des Bürgertums in Warschau allenfalls noch als deutschstämmig, waren die Lódzer Unternehmer noch deutscher, was sich seit 1906 auch an einem „Hilfsverein der deutschen Reichsangehörigen“ mit alldeutschen Zielen ablesen lässt.

Wie stark ökonomische Interessengegensätze innerhalb derselben sozialen Schicht ethnisch und national aufgeladen werden konnten, zeigt die wirtschaftspolitische Polemik, als Moskauer Vertreter der Textilindustrie zwischen Kongresspolen und Russland wieder Zollbarrieren aufstellen wollten und zudem den russischen Patriotismus der Lódzer Industriellen in Frage stellte. Journalisten aus St. Petersburg und Moskau beschworen die „deutsche Gefahr" herauf und sahen in Lódzer Fabrikanten willfährige Organe der Regierungszentrale von Berlin. In einer öffentliche Rede von 1885 versuchte ein Interessenvertreter der Moskauer Unternehmerschaft namens Sharapow aus 
dem Konkurrenzkampf zwischen Moskau und Lódz einen rassischen Zweikampf zwischen Slawen- und Germanentum zu machen (Luxemburg 1898: 154-159). Er rief die Regierung zum Kampf gegen die „deutsche“ Industrie Polens und zur Rettung des von ihr unterdrückten russischen und polnischen Elements auf, ungeachtet dessen, dass gerade die Polen am meisten unter der Zwangsherrschaft des Zaren zu leiden hatten. Die russische Regierung entsandte im Jahre 1886 eine dreiköpfige Regierungskommission nach Lódz, um dem Erfolgsgeheimnis der dortigen Industrie auf die Spur zu kommen. In der 1888 in St. Petersburg veröffentlichten Expertise unter dem Titel „Bericht über die Untersuchung der Fabrikindustrie im Zartum Polen“ kam sie zu dem Ergebnis, dass die Lódzer Industriellen die Kohle billiger einkauften als deren Moskauer Kollegen, die aber dafür den Arbeitern geringere Löhne auszahlten. Wenn auch die Lódzer Bourgeoisie weniger Steuern entrichtete, so würde sie andererseits mehr auf dem Gebiet der sozialen Fürsorge leisten. Die Kommission wunderte sich darüber, dass es im Lande geborene „russische“ Staatsbürger gebe, die kaum des Russischen mächtig seien (Heike 1971: 236). Letztlich wurde allen Beteiligten dieses Streits bewusst, dass mit ethnischer Propaganda den industriellen Geschäftsinteressen am wenigsten gedient war.

Das Verhältnis der Lódzer Unternehmer gegenüber nationalen Bindungen und Verpflichtungen blieb von einem großen Pragmatismus geprägt. Wenn sich die deutschstämmigen Fabrikanten auch in den wenigsten Fällen offen antipolnisch zeigten, waren viele doch obrigkeitstreu genug eingestellt, nach der niedergeschlagenen Revolution von 1864 Glückwunschadressen an den Zaren zu schicken. Loyal gegenüber der russischen Herrschaft ging man ohne nationale Erwägungen ${ }^{1}$ seinen geschäftlichen Interessen nach. Die Nationalität gewann allenfalls unter ökonomischen Gesichtspunkten an Bedeutung: Russische Gesetze schufen Anreize zur Annahme der russischen Staatsangehörigkeit, da Ausländern die Bildung von Aktiengesellschaften verboten war (Rogall 1998: 444).

Bis zum Ersten Weltkrieg waren Familien wie die Grohmanns, Geyers, Hoffrichters zum großen Teil polonisiert. Die zweite Generation Geyers war schon in verschiedenen polnischen Vereinen engagiert. Wenn auch in den neunziger Jahren beim Führungspersonal der Lódzer Betriebe, d.h. bei Meistern und technischem Personal mehr als die Hälfte Deutsche waren, holten hier die Polen immer mehr auf (Pytlas 1994: 299). Schon der aus einer Lódzer Fabrikantenfamilie stammende Kurt Schweikert benutzte den Begriff „polnische Bourgeoisie“ und meinte damit sein multinationales Bürgertum (Schweikert 1913). 1926 wurde die Organisiation der Evangelischen Polen ge-

1 Zwei Söhne des Fabrikanten Steinert besaßen die deutsche, die anderen beiden die russische Staatsangehörigkeit. Von den Söhnen Karl Scheiblers war der eine im Ersten Weltkrieg russischer, der andere deutscher Offizier. 
gründet, der auch die ursprünglich deutschsprachigen Industriellen Geyer oder Grohmann angehörten.

\section{VII.}

Auf der Jagd nach möglichst großem kapitalistischen Gewinn wirkte die Rücksichtnahme auf konfessionelle oder auch nationale Interessen wie ein Relikt aus einer vergangenen Zeit. Gerade weil sie im Geschäftsleben ihre ethnische Herkunft weitgehend vergaßen und sich auf die neue Umgebung pragmatisch einstellten, verwandelte sich die Spitze der Großindustrie von Lódz zu erfolgreichen Unternehmern und zu den reichsten Bürgern in Polen. Um die Jahrhundertwende sollen vierzig Lódzer Unternehmer ein Vermögen von über eine Million Rubel besessen haben (Pytlas 1994: 57f). Die Protestanten Geyer, Scheibler und Grohmann sind ebenso zu nennen wie die katholische Familie Heinzel. Alfred Döblin beschrieb in seinem Buch „Reise in Polen“"von 1926 das deutsch-polnische Zusammenleben in Lódz und betonte einen Zusammenhang zwischen Wohlstand und Assimilationsfähigkeit:

„Der Patriotismus wächst also mit dem Geldschwund? Nein. Ein Armer hat einfach Not; die macht kämpferisch, verhindert Kompromisse. Der Reiche aber will etwas für sein Geld und nimmt auch fremden Glanz: Geld contra Nationalität“ (in: Heike 1971: 62f.).

Ein nicht unerheblicher Anteil der deutschsprachigen, katholischen Fabrikanten, der meist aus Böhmen oder Schlesien stammte, verstand es, sich rasch zu polonisieren. Der 1834 als Sohn schlesischer Einwanderer geborene Julius Heinzel, der gemeinsam mit Julius Kunitzer im Dorf Widzew eine Fabrik anlegte, aus der 1884 eine Aktiengesellschaft für Baumwollerzeugnisse hervorging, konnte daher sagen:

„Mein Vater sprach nur schlecht polnisch. Mein Bruder und ich sind bereits Polen, und außerdem sind ja hier heutzutage alle schon mehr Polen als Deutsche“ (zitiert in: Gorski 1904: 21f.).

Beschäftigt man sich mit der sozialen Elite der Unternehmer, bestand die Besonderheit von Lódz weniger in der ethnischen Potenzierung des allgegenwärtigen sozialen Gegensatzes - ein journalistischer Beobachter beschrieb die revolutionären Ereignisse zwischen 1905 und 1907 mühelos als Kampf zwischen polnischen Arbeitern und einer ihr ethnisch fremden Bourgeoisie (Bartkiewicz 1911: 5) - als vielmehr darin, dass im Gegensatz zu Deutschland, wo der Spielraum der einzelnen Unternehmer durch kollektive Regelungen und Prozesse der Verrechtlichung und Bürokratisierung zunehmend eingeschränkt war, die Unternehmer in Lódz freier agieren konnten und ein von Interventionen einer weit entfernten Regierung unbehelligtes Geschäftsleben führen konnten, in dem ethnische und nationale Interessen nur am Rande ihren Einfluss geltend machten. Nur so konnte mit der auf deutsch so genannten zeitgenössichen Kategorie des „Lódzer Menschen“ - fest im polnischen und jiddischen Wortschatz verankert - in der Phase des Hochkapitalismus eine eigene, „staatenlose Nationalität“ entstehen: 
„Ursprünglich war Deutschland ihr Vaterland; doch ihr längerer, seit mehreren Generationen andauernder Aufenthalt in unserem Lande ließ schließlich viel von ihrem germanischen Patriotismus schwinden, brachte sie aber der polnischen Nationalität noch immer nicht näher. Hauptsächlich handelt es sich bei ihnen um Menschen ohne politische Überzeugung" (ebd).

Literaturnobelpreisträger Wladyslaw Reymont machte in seinem Lódzer Roman „Ziema Obiecana“ von 1898, der 1974 von Andrzej Wajda verfilmt werden sollte, die ethnisch-kulturellen Spannungen zwischen polnischen, jüdischen und deutschen Unternehmern vor dem Hintergrund einer der Ausbeutung preisgegebenen, insbesondere aus Polen bestehenden Arbeiterschaft zum Thema (Rhode 1960). Er sah in dem Lódzer Menschen eine seelen- und kulturlose Kreatur und verglich ihn in seinem Drang nach Profit mit einer rastlosen Maschine, der sich als skrupelloser Egoist während der rasanten Entwicklung des Kapitalismus nur um seine Geschäfte kümmern würde. Tatsächlich hielten viele Geschäftemacher, die es unter deutsch-jüdischem Einfluss zu etwas gebracht hatten, wenig von Nation und Politik. Stattdessen verkörperten sie einen anpassungsfähigen, meist aber auch opportunistischen dreisprachigen „Rubelpatriotismus“ (Breyer 1978). Wenn ein längerfristiger ethnischer Identitätsbildungsprozess bemerkbar war, dann war es die aus der Bevölkerungsentwicklung natürlich zu erklärende, nie reibungslos sich vollziehende Polonisierung, die vom nationalsozialistischen Terror abrupt gestoppt werden sollte: Am 5. Juni 1997 wurde an der „weißen Fabrik“, dem ehemaligen Firmensitz der Familie Geyer eine Gedenktafel angebracht, die an die Ermordung eines Polen deutscher Herkunft namens Robert Geyer durch die Gestapo im Dezember 1939 erinnert, der sich geweigert hatte, sich zum Deutschtum zu bekennen und daher die Volksliste nicht unterschrieb.

\section{Literatur}

Bartkiewicz, Zygmunt (1911): Zle miasto. Obrazy z 1907 r., Warszawa.

Bielschowsky, Frieda (1912): Die Textilindustrie des Lódzer Rayons. Leipzig.

Breyer, Richard (1978): Der Lódzer Mensch - Legende und Wirklichkeit, in: Peter Nagarski (Hg.), Lódz - die Stadt der Völkerbegegnung im Wandel der Geschichte, Köln, 74-76.

Felde, Léon (1935): Lódz. Le Manchester Polonais, Lódz.

Friedman, Filip (1935): Dzieje Zydow w Lódzi od poczatkow osadnictwa Zydow do r. 1863, Lódz.

Gasiorowska, Natalia (1922): Osadnictwo fabryczne, in: Ekonomista 22 (1922), 140 und 111-151.

Gorgolowski, Jozef (1985): Poznanski, Izrael (1833-1900), in: Polski Slownik Biograficzny 28, 293-295.

Gorski, S. (1904): Lódz spolczesna. Obrazki i skice publicytyczne, Lódz.

Guesnet, Francois (1999): Das Verhältnis von Juden und Deutschen im Spiegel ihrer Organisationen im Lódz des 19. Jahrhunderts, in: Jürgen Hensel (Hg.), Polen, Deutsche und Juden in Lódz 1820-1939. Eine schwierige Nachbarschaft, Osnabrück, 139-171.

Heike, Otto (1971): Aufbau und Entwicklung der Lódzer Textilindustrie: Eine Arbeit deutscher Einwanderer in Polen für Europa, Mönchengladbach.

Janczak, J. (1982): Ludnosc Lódzi przemyslowej 1820-1914, Lódz.

Janowicz, Ludwik (1907): Zarys rozwoju premyslu w Krolestwie polskim, Warszawa.

Kaczynska, Elzbieta (1988): Bürgertum und städtische Elite. Kongreßpolen, Rußland und Deutschland im Vergleich, in: Jürgen Kocka, Bürgertum im 19. Jahrhundert, München, 466-489.

KoLódziejczyk, Ryszard (1986): Die Bourgeoisie im Königreich Polen. Entwurf eines Porträts, in: Scripta Mercaturae 20, 58-77. 
Kula, W. (1955): Ksztaltowanie sie kapitalizmu w Polsce, Warszawa.

Luxemburg, Rosa (1898): Die industrielle Entwicklung Polens - Inaugural-Dissertation zur Erlangung der Staatswissenschaftlichen Doktorwürde an der Universität Zürich, Leipzig 1898, [in: Rosa Luxemburg, Gesammelte Werke, Band 11893 bis 1905, 1. Halbband, Berlin 1982, 113216].

McKay, John P. (1970): Pioneers for Profit. Foreign Entrepreneurship and Russian Industrialization, 1885-1913, Chicago.

Mosse, Werner E. (1992): Jewish Entrepreneurship in Germany 1820-1935, in: ders. / Hans Pohl (Hg.), Jüdische Unternehmer in Deutschland im 19. und 20. Jahrhundert, Stuttgart, 54-67.

Pastula, Zbigniew (1994): Scheibler, Karol W. (1820-1881), in: Polski Slownik Biograficzny 35, 426430.

Pietrow-Ennker, Bianka (1999): Auf dem Weg zur Bürgergesellschaft. Modernisierungsprozesse in Lódz (1820-1914), in: Jürgen Hensel (Hg.), Polen, Deutsche und Juden in Lódz 1820-1939. Eine schwierige Nachbarschaft, Osnabrück, 103-131.

Pytlas, Stefan (1994): Lódzka burzuazja przemyslowa 1864-1914, Lódz.

Rogall, Jochen (1998): Deutsche Unternehmer im Lódzer Raum, in: Dittmar Dahlmann / Carmen Scheide (Hg.) „... das einzige Land in Europa, das eine große Zukunft vor sich hat.“ Deutsche Unternehmen und Unternehmer im Russischen Reich im 19. und frühen 20. Jahrhundert, Essen, 437-453.

Rhode, Gotthold (1960): Das Bild des Deutschen im polnischen Roman des 19. und beginnenden 20. Jahrhunderts und das polnische Nationalgefühl, in: Ostdeutsche Wissenschaft. Festgabe für Max Hildebert Boehm, München, 327-366.

Rose, E. (1918): Wielki przemysl Krolestwa Polskiego przed wojna. przyczynki do tzw. „teorii rynkow wschodnich", Poznan.

Rozanski, A (1948): Proba okreslenia liczby imigrantow niemieckich przybylych na teren Krolestwa Polskiego, in: Roczniki Dziejow Spolecznych i Gospodarczych 10, $200 \mathrm{ff}$.

Samus, Pawel (1999): Lódz, Heimatstadt von Polen, Deutschen und Juden, in: Jürgen Hensel (Hg.), Polen, Deutsche und Juden in Lódz 1820-1939. Eine schwierige Nachbarschaft, Osnabrück, 13-31.

Schumpeter, Jospeph (1920): Unternehmer, in: Handwörterbuch der Staatswissenschaften 8, 476-487.

Schweikert, Kurt (1913): Die Baumwollindustrie Russisch-Polens, Zürich/ Leipzig.

Sombart, Werner (1902): Der moderne Kapitalismus. Historisch-systematische Darstellung des gesamteuropäischen Wirtschaftsleben von den Anfängen bis zur Gegenwart, Berlin.

Strobel, Georg W. (1992): Lódz: eine Vielvölkerstadt Polens, in: Deutsche und Polen. 100 Schlüsselbegriffe, hg. von E. Kobylinska u.a., München, 510f.

Thompson, Edward P. (1968): The Making of the English Working Class, Harmondsworth.

Tych, Feliks (1999): Lódzer Polen, Juden und Deutsche in der Revolution von 1905, in: Jürgen Hensel (Hg.), Polen, Deutsche und Juden in Lódz 1820-1939. Eine schwierige Nachbarschaft, Osnabrück, 199-215.

Waskiewicz, Ludwik (1958): Geyer, Ludwik (1805-1869), in: Polski Slownik Biograficzny 7, 414f.

Walter, Rolf (1992): Jüdische Unternehmer in der deutschen Textilindustrie bis 1932, in: Werner E. Mosse / Hans Pohl (Hg.), Jüdische Unternehmer in Deutschland im 19. und 20. Jahrhundert, Stuttgart, 132-153 